\title{
Nature Plus Plus Inspired Computing - The Superset of Nature Inspired Computing
}

Abstract- The term "Nature Plus Plus Inspired Computing" is coined by us in this article. The abbreviation for this new term is " $\mathrm{N}++\mathrm{IC}$." Just like the $\mathrm{C}++$ programming language is a superset of $\mathrm{C}$ programming language, Nature Plus Plus Inspired Computing $(\mathrm{N}++\mathrm{IC})$ field is a superset of the Nature Inspired Computing (NIC) field. We defined and introduced "Nature Plus Plus Inspired Computing Field" in this work. Several interesting opportunities in N++IC Field are shown for Artificial Intelligence Field Scientists and Students. We show a literature review of the $\mathrm{N}++$ IC Field after showing the definition of Nature Inspired Computing (NIC) Field. The primary purpose of publishing this innovative article is to show a new path to NIC Field Scientists so that they can come up with various innovative algorithms from scratch. As the focus of this article is to introduce $\mathrm{N}++\mathrm{IC}$ to researchers across the globe, we added $\mathrm{N}++\mathrm{IC}$ Field concepts to the Particle Swarm Optimization algorithm and created the "Children Cycle Riding Algorithm (CCR Algorithm)." Finally, results obtained by CCR Algorithm are shown, followed by Conclusions.

Keywords: nature inspired computing, nature plus plus inspired computing, artificial intelligence, children, evolutionary computing, computational intelligence, new area, interesting opportunities, children cycle riding, children swarm.

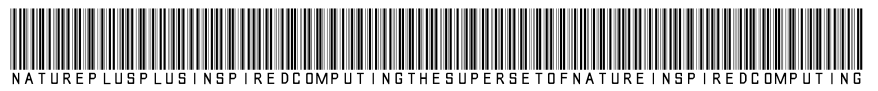

Strictly as per the compliance and regulations of:

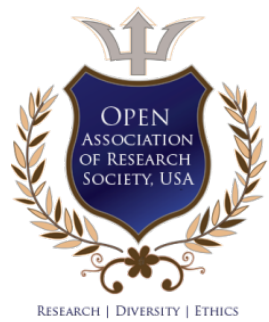

(C) 2020. Satish Gajawada \& Hassan M. H. Mustafa. This is a research/review paper, distributed under the terms of the Creative Commons Attribution-Noncommercial 3.0 Unported License http://creativecommons.org/licenses/by-nc/3.0/), permitting all non commercial use, distribution, and reproduction in any medium, provided the original work is properly cited. 


\title{
Nature Plus Plus Inspired Computing - The Superset of Nature Inspired Computing
}

\author{
Satish Gajawada ${ }^{\alpha}$ \& Hassan M. H. Mustafa $^{\sigma}$
}

Abstract- The term "Nature Plus Plus Inspired Computing" is coined by us in this article. The abbreviation for this new term is "N++IC." Just like the $\mathrm{C}++$ programming language is a superset of $\mathrm{C}$ programming language, Nature Plus Plus Inspired Computing $(\mathrm{N}++\mathrm{IC})$ field is a superset of the Nature Inspired Computing (NIC) field. We defined and introduced "Nature Plus Plus Inspired Computing Field" in this work. Several interesting opportunities in $\mathrm{N}++\mathrm{IC}$ Field are shown for Artificial Intelligence Field Scientists and Students. We show a literature review of the $\mathrm{N}++\mathrm{IC}$ Field after showing the definition of Nature Inspired Computing (NIC) Field. The primary purpose of publishing this innovative article is to show a new path to NIC Field Scientists so that they can come up with various innovative algorithms from scratch. As the focus of this article is to introduce $\mathrm{N}++\mathrm{IC}$ to researchers across the globe, we added $\mathrm{N}++\mathrm{IC}$ Field concepts to the Particle Swarm Optimization algorithm and created the "Children Cycle Riding Algorithm (CCR Algorithm)." Finally, results obtained by CCR Algorithm are shown, followed by Conclusions.

Keywords: nature inspired computing, nature plus plus inspired computing, artificial intelligence, children, evolutionary computing, computational intelligence, new area, interesting opportunities, children cycle riding, children swarm.

\section{Contribution of Authors:}

1. Direct Contribution: Hassan M. H. Mustafa and Satish Gajawada made a direct and complete contribution to this article. They have contributed equally to this article.

\section{Definition of New Nature Plus Plus Inspired Computing Field}

$\mathrm{N}$ ature Inspired Computing (NIC) Algorithms take inspiration from Mother Nature. Nature Inspired Computing Algorithms are a subset of Nature Plus Plus Inspired Computing $(\mathrm{N}++\mathrm{IC})$ Field Algorithms. Hence an algorithm belonging to the NIC field also belongs to the $\mathrm{N}++\mathrm{IC}$ field. If an algorithm takes inspiration from Artificial things in addition to inspiration taken from nature, then such algorithms belong to both $\mathrm{NIC}$ and $\mathrm{N}++\mathrm{IC}$ fields. Also, there can be algorithms that can take inspiration completely from

Author $\alpha$ : Independent Inventor and Scientist. Alumnus, Indian Institute of Technology Roorkee. The Creator of Artificial Satisfaction Field. Founder and Father of Artificial Human Optimization Field. Inventor of Deep Loving Field. Designer of Nature Plus Plus Inspired Computing Field. Inventor of Artificial Soul Optimization and Artificial God Optimization Fields. e-mail: satish.gajawada.iit@gmail.com

Author o: Banha University, Egypt.
Artificial things, and there is no inspiration taken from nature, then such algorithms belong only to the $\mathrm{N}++\mathrm{IC}$ field and not the NIC field. There are three types of algorithms. Algorithms that take inspiration from nature only. The second type of algorithms are such that they take inspiration only from artificial things. The third type of algorithms takes inspiration from both nature as well as artificial things. The first category of algorithms belongs to $\mathrm{NIC}$. The second category of algorithms belongs only to the $\mathrm{N}++\mathrm{IC}$ field and not the NIC field. The third category of algorithms belongs to both NIC and $\mathrm{N}++\mathrm{IC}$ fields. All three types of algorithms belong to the $\mathrm{N}++\mathrm{IC}$ field. In the $\mathrm{N}++\mathrm{IC}$ field, we added one more type of algorithms in addition to NIC field algorithms. Hence NIC field is a subset of the $\mathrm{N}++\mathrm{IC}$ field.

\section{il. Interesting Opportunities in Nature Plus Plus Inspired Computing Field}

There are INTERESTING OPPORTUNITIES for NATURE INSPIRED COMPUTING (NIC) field Research Scientists in NATURE PLUS PLUS INSPIRED COMPUTING $(\mathrm{N}++\mathrm{IC})$ field. Some of them are shown below:

1. B.Tech Project in $\mathrm{N}++\mathrm{IC}$ field, IIT Roorkee

2. M.Tech Project in $\mathrm{N}++\mathrm{IC}$ field, Harvard University

3. Ph.D. in $\mathrm{N}++\mathrm{IC}$ field, IIT Hyderabad

4. Postdoc in $\mathrm{N}++\mathrm{IC}$ field, Stanford University

5. International Association of $\mathrm{N}++\mathrm{IC}$ field, Singapore

6. International Conference on $\mathrm{N}++\mathrm{IC}$ field, Dubai

7. Transactions on NIC and $\mathrm{N}++\mathrm{IC}$, United Kingdom

8. International Journal on $\mathrm{N}++\mathrm{IC}$ field, Australia

9. International Workshop on $\mathrm{N}++\mathrm{IC}$ field, Hong Kong

10. The foundation on $N++I C$, New York

11. Seminar on $N++I C$ field at Technical Festival in Pakistan colleges

12. Microsoft R\&D team on $\mathrm{N}++\mathrm{IC}$ field

13. IBM R\&D N++IC field Research Labs, IBM Hyderabad

14. YouTube videos on N++IC and NIC fields by Google R\&D team, Google Delhi

15. Springer Journal on $\mathrm{N}++\mathrm{IC}$

16. Elsevier book on $\mathrm{N}++\mathrm{IC}$

17. IEEE $\mathrm{N}++$ IC Society, Japan

18. To become a Scientist in the $\mathrm{N}++\mathrm{IC}$ field

19. A Course on $\mathrm{N}++\mathrm{IC}$ by Coursera 
20. Advanced $\mathrm{N}++\mathrm{IC}-\mathrm{A}$ New subject

21. IBMSUR Award for a Professor in $\mathrm{N}++$ IC FIELD at IIT Hyderabad

\section{ili. Nature Inspired Computing}

According to [1], the definition of NATURE INSPIRED COMPUTING is shown below in doublequotes as it is:

"The field of nature-inspired computing (NIC) is interdisciplinary in nature combining computing science with knowledge from different branches of sciences, e.g. physics, chemistry, biology, mathematics and engineering, that allows development of new computational tools such as algorithms, hardware, or wetware for problem-solving, synthesis of patterns, behaviours and organisms."

\section{Literature Review}

There are many Research Scientists and Students who are working in the field of Nature Inspired Computing. You will easily find thousands of references for Nature Inspired Computing when you search on Google. In this paper, our focus is to define a new field titled Nature Plus Plus Inspired Computing $(\mathrm{N}++\mathrm{IC})$ and how it is related to Nature Inspired Computing (NIC). Hence for the sake of completeness, we just show [1] [10] articles that come under NIC. As defined, NIC is a subset of $\mathrm{N}++\mathrm{IC}$, and hence all [1] - [10] articles also belong to the $\mathrm{N}++\mathrm{IC}$ field.

\section{Children Cycle Riding Algorithm}

Figure 1 shows the Children Cycle Riding Algorithm (CCRA). In this section, we explain CCRA. In the beginning, the iteration counter is set to zero, and all Artificial Children are initialized. The search space is full of Artificial sharp stones, which may result in damaging the tyre of the Artificial Child's Cycle. Hence we have Cycle Tyre Damage Probability. After the damage of the cycle tyre, the child repairs his cycle tyre with probability Cycle Tyre Repaired Probability.

If a cycle tyre is damaged, then there are two possibilities. Either Artificial Child repairs his cycle tyre or not. If the cycle tyre is damaged and Artificial Child gets his cycle repaired, then Artificial Child can move in search space and hence updates Velocity and Position. If the cycle tyre is damaged and Artificial Child cannot repair his cycle tyre then Artificial Child is halted and does not update his Velocity and Position. On the other hand, if Artificial Child's Cycle tyre is not damaged, then he can move in search space and hence updates Velocity and Position. At the end of the iteration, the iteration counter is incremented. Now the control goes to line number 2. This process is continued until the termination condition is reached. Figure 1 is shown below:
1) All Artificial Children are initialized, and the iteration counter is set to zero.
2) Artificial Children identifies their local best
3) Artificial Children identifies their global best
4) Artificial Children identifies their local worst
5) Artificial Children identifies their global worst
6) for each Artificial Child do
7) if ( random $(0,1)<$ CycleTyreDamageProbability) then
8) if ( random $(0,1)<$ CycleTyreRepairedProbability) then
9) Artificial Child updates Velocity
10) Artificial Child updates Position
11) else
12) // If Cycle tyre is damaged and it is not repaired then Artificial Child is $/ /$ halted and does nothing
13) end if
14) else
15) Artificial Child updates Velocity
16) Artificial Child updates Position
17) end if
18) end for
19) Update Iteration Counter
20) if (termination_condition_reached is not true) then
21) jump to line number 2
22) end if

Figure 1: Children Cycle Riding Algorithm (CCRA) 


\section{Results}

The Human Poverty Particle Swarm Optimization (HPPSO) proposed in [11], and Children Cycle Riding Algorithm (CCRA) proposed in this article are MATHEMATICALLY EQUAL. In [11], it was shown that both HPPSO and PSO performed well on all benchmark functions. Hence due to Mathematical EQUALITY, both CCRA and PSO Algorithms performed well on all benchmark functions.

\section{Vil. Conclusions}

"Nature Plus Plus Inspired Computing $(\mathrm{N}++\mathrm{IC})$ " field is designed and introduced in this work. The difference between the two fields NIC and the $\mathrm{N}++\mathrm{IC}$ is clearly explained. Children Cycle Riding Algorithm (CCRA) is designed, and results show that CCRA performed as good as the Particle Swarm Optimization algorithm. Some interesting opportunities in the $\mathrm{N}++\mathrm{IC}$ field are shown for NIC field Students and Research Scientists. Research Scientists and Students did a lot of research in the NIC field. There is a lot of scope in the direction where Algorithms are inspired by both nature and Artificial things. Also, there exists a lot of scope in the direction where Algorithms are inspired by Artificial things only. Children are natural, and cycle riding is Artificial. Hence CCRA is designed by taking inspiration from both nature and Artificial things. This paper is mainly published to introduce $\mathrm{N}++\mathrm{IC}$ Field to the world. Hence we just added $\mathrm{N}++\mathrm{IC}$ concepts to the Particle Swarm Optimization algorithm and created CCRA. As the new field is proposed in this article, the next step for Researchers is to create new $\mathrm{N}++\mathrm{IC}$ field Algorithms from scratch.

\section{ACKNOWLEDGMENTS}

Thanks to the strong editorial team of GJCST and reviewers for accepting our innovative inventon titled "N++IC Field". Thanks to everyone (and everything) who directly or indirectly helped us to reach the stage where we are now today.

\section{References Références Referencias}

1. Nazmul Siddique and Hojjat Adeli. "Nature Inspired Computing: An Overview and Some Future Directions". Cognitive Computation. 2015. Springer.

2. Lei Wang, Qi Kang, Qi-di Wu, Nature-inspired Computation - Effective Realization of Artificial Intelligence, Systems Engineering - Theory and Practice, Volume 27, Issue 5, 2007.

3. Alexandros Tzanetos, Iztok Foster, Georgios Dounias. A comprehensive database of NatureInspired Algorithms, Data in Brief, Volume 31, 2020.

4. Marrow, P. Nature-Inspired Computing Technology and Applications. BT Technology Journal 18, 13-23 (2000)
5. Sergeyev, Y.D., Kvasov, D.E. \& Mukhametzhanov, M.S. On the efficiency of nature-inspired metaheuristics in expensive global optimization with limited budget. Sci Rep 8, 453 (2018).

6. Shruti Goel, Manas Gaur, Eshaan Jain. Nature Inspired Algorithms in Remote Sensing Image Classification. Third International Conference on Recent Trends in Computing 2015 (ICRTC - 2015).

7. Xin-She Yang, Nature-Inspired Optimization Algorithms: 2nd Edition, Elsevier, (2020).

8. Ashraf Darwish, Bio-inspired computing: Algorithms review, deep analysis, and the scope of applications, Future Computing and Informatics Journal, Volume 3, Issue 2, 2018.

9. Binitha S, S Siva Sathya. A Survey of Bio inspired Optimization Algorithms. International Journal of Soft Computing and Engineering (IJSCE). Volume 2, Issue 2, 2012.

10. Crowcroft J. (2008) Bio-Inspired Computing and Communication. Lecture Notes in Computer Science, vol 5151. Springer, Berlin, Heidelberg.

11. Satish Gajawada, Hassan M. H. Mustafa. Ten Artificial Human Optimization Algorithms. Transactions on Machine Learning and Artificial Intelligence. Volume 7, № 3. 2019. 